\title{
Implementación de cultivos hidropónicos como estrategia pedagógica para el fomento de la investigación ${ }^{1}$
}

\author{
Implementation of hydroponic crops as a pedagogical \\ strategy for the promotion of research
}

DOI: http://dx.doi.org/10.17981/cultedusoc.9.3.2018.43

Artículo de investigación. Fecha de recepción: 15/06/2018. Fecha de aceptación: 27/11/2018

\begin{abstract}
Faber Mejía; Jaime Pérez; Bladimiro Ricaurte; Catalino Urieles; Clelia Melo Beltrán; Danellis Bolaño; Farides García; Gasalis Córdoba; Iliana Carretero; Jaider Barraza; Jairo Quintero; Juan Pineda; Libia López; Luis Pérez; María Flores; Mercy Mejía; Miriam Velaider; Raúl Rocha Comas; Julia Rodríguez; Marina Márquez Martínez; Orlaida Torres; Yulis García; Rita Barraza; José De Jesús Blanco; Leonid De León; Yarley Acuña y Pilar Guerra ${ }^{2}$

Institución Educativa Departamental Centro de Educación Básica San Valentín (Colombia)
\end{abstract}

Para citar este artículo:

Mejía, F., Pérez, J. Ricaurte, B., Urieles, C., Melo, C., Bolaño, D., García, F., Córdoba, G., Carretero, I., Barraza, J., Quintero, J., Pineda, J., López, L, Pérez, L., Flores, M., Mejía, M., Miriam Velaider; Raúl Rocha Comas; Julia Rodríguez; Marina Márquez Martínez; Orlaida Torres; Yulis García; Rita Barraza; De Jesús, J., De León, L., Acuña, Y. y Guerra, P. (2018). Implementación de cultivos hidropónicos como estrategia pedagógica para el fomento de la investigación. Cultura. Educación y Sociedad 9(3), 377-382. DOI: http://dx.doi. org/10.17981/cultedusoc.9.3.2018.43

\section{Resumen}

En el presente proyecto se desarrollaron actividades pedagógicas para el fortalecimiento de los procesos educativos en la población estudiantil de la institución: Centro de Educación Básica San Valentín, en el departamento del Magdalena, a través de implementación de cultivos hidropónicos, como estrategias pedagógicas que desarrollan habilidades investigativas en infantes y jóvenes de la institución. Se trabajó bajo la metodología cualitativa con modelo de investigación acción participativa. La población estuvo conformada por estudiantes de esa institución. Se recogió la información por medio de las técnicas como relatorías individuales, institucionales, diario de campo, encuestas y observación directa. Se identificaron los contenidos curriculares del área de Biología para aplicar en los cultivos como un recurso generador de aprendizaje, con base en los cuales se realizó la planeación para las clases y el trabajo en los cultivos.

Palabras clave: Cultivos hidropónicos, estrategia pedagógica, investigación

\section{Abstract}

In the present project, pedagogical activities were developed for the strengthening of educational processes in the student population of the San Valentín Basic Education Center, in the department of Magdalena, through the implementation of hydroponic crops as pedagogical strategies that develop investigative skills in infants and young people of the institution. We worked under the qualitative methodology with participatory action research model. The population was conformed by students of the San Valentín Basic Education Center, in Magdalena. The information was collected by means of techniques such as individual and institutional rapporteurships, field journals, surveys and direct observation. The curricular contents of the Biology area to be applied in the crops were identified as a learning instrument, based on which the planning for the classes and the work in the crops was carried out.

Keywords: Hydroponic crops, pedagogical strategy, research

1 Este artículo ha sido derivado del Proyecto Fortalecimiento de la Cultura Ciudadana y Democrática en CT+I a través de la IEP apoyada en TIC en el Dpto. del Magdalena.

2 Docentes de la institución Educativa Departamental Centro de Educación Básica San Valentín

- The author; licensee Universidad de la Costa - CUC.

Cultura, Educación y Sociedad vol. 9 no. 3, pp. 377-382. Diciembre, 2018

Barranquilla. ISSN 2389-7724 Online 


\section{Introducción}

Los cultivos hidropónicos como estrategia pedagógica que fomenta la investigación en los estudiantes, orienta hacia la búsqueda de opciones viables para generar nuevos conocimientos y al mismo tiempo brindar herramientas a los estudiantes para su sustento, ofreciendo a las familias una forma práctica de ser productivos sin tener extensiones de terreno, con la misión de mejorar las condiciones de vida de los niños, niñas, jóvenes y adultos y de la comunidad en general.

Las demandas sociales y económicas en el contexto de la Institución Educativa Departamental Rural San Valentín, conducen a la necesidad de revisar el quehacer educativo. Existen tipos de aprendizajes importantes, entre los cuales se menciona, aprender a conocer, aprender a hacer, aprender a convivir y aprender a ser (Delors, 1994; Cajiao \& Uribe, 2016). Este segundo aspecto es muy significativo para la comunidad que rodea a la institución educativa, debido a los cambios y las necesidades presentes, las cuales incluyen escasez de los recursos naturales, deterioro ambiental, pérdida de la biodiversidad, dificultad en la repartición y tenencia de la tierra; además de los desbalances económicos y sociales, por solo mencionar algunas de las dificultades.

Teniendo en cuenta esto, surge la necesidad de implementar estrategias consecuentes con la problemática social emergente; el uso de cultivos hidropónicos hace referencia al cultivo en agua, en contraposición al tradicional cultivo en suelo (Beltrano \& Gimenez, 2015; Fontán, 2016; Grijota, 2017; Arquigráfico, 2018), lo cual permite a los estudiantes por medio de la investigación como estrategia pedagógica, conocer alternativas que permitirán ampliar conocimientos aplicables a su realidad. Es decir, la estrategia pedagógica para la implementación de cultivos hidropónicos, genera conocimientos necesarios en los educandos de la institución educativa San Valentín, y a su vez, esta tarea educativa de carácter agrícola contribuye con la formación de actitudes y valores, de una generación desasistida por los diferentes actores sociales de este municipio y responde acertadamente a las demandas del entorno, tales como mejorar la producción de alimentos para la población estudiantil.

En base a lo anterior, se plantea este proyecto de investigación dirigido a la población estudiantil de la institución Centro de Educación Básica San Valentín, en el Magdalena en el marco de la Investigación como Estrategia Pedagógica (IEP) la cual, reconoce en el ejercicio de la investigación en los procesos de aprendizaje como una forma de cambiar concepciones, realidades y el entorno de los participantes, y con ello una manera de situarse crítica y éticamente en el mundo cercano, mediato y global (Mejía y Manjarréz, 2011; Villalba, 2012; Orozco, 2015; Restrepo, 2009).

\section{Metodología}

El artículo que se presenta es producto del estudio sobre la implementación de cultivos hidropónicos como estrategia pedagógica para el fomento de la investigación. Desarrollado en la institución Centro de Educación Básica San Valentín; su objetivo principal ha sido implementación de cultivos hidropónicos como estrategia pedagógica, que favorece el desarrollo de habilidades investigativas.

El estudio contempla como procesos metodológicos los siguientes: El diseño elegido fue dirigido bajo la modalidad de investigación cualitativa, porque estudia la realidad en su contexto natural, tal y como sucede, intentando extraer sentido de, o interpretar los fenómenos, de acuerdo con los significados que tienen para las personas implicadas (Ruiz, 2012; Pérez, 2002; Hernández 2014) Se utilizó el modelo de investigación acción participativa, porque 
es un procedimiento que forma parte de una estrategia que considera a la comunidad en la construcción del conocimiento y solución de sus problemas, en este sentido, no busca solamente describir los problemas sino, generar conjuntamente con la comunidad los conocimientos necesarios para definir las acciones adecuadas que estén en la línea del cambio y la transformaciones necesarias. (Báez, 2009; Martínez, 2004).

La unidad de análisis utilizada estuvo conformada por estudiantes de la institución Centro de Educación Básica San Valentín, en el Magdalena. El instrumento empleado para la recolección de información, fueron técnicas como relatorías individuales, institucionales, diario de campo, encuestas y observación directa.

\section{Resultados}

- Se identificaron los contenidos curriculares del área de Biología a aplicar en los cultivos como un instrumento generador de aprendizaje.

- Se identificaron los contenidos y competencias específicas a desarrollar en el área de Biología, con base en los cuales se procedió a realizar la planeación para las clases y el trabajo en los cultivos.

- Se logró identificar las diferentes técnicas de cultivos hidropónicos aplicables en el contexto institucional, así como las variedades de hortalizas cultivables a través de la hidroponía.

- Se logró establecer un sistema hidropónico escolar para mejorar la calidad de las variedades cultivadas.

- Se mejoró la calidad de las hortalizas que consumen las familias de la comunidad educativa.

\section{Conclusiones}

Se concluye que el alcance de los conocimientos agrícolas debe estar articulado con la aplicación de estrategias pedagógicas, que den como resultado estudiantes con conocimientos amplios sobre las prácticas agrícolas y por ende, el docente se convierte en un verdadero facilitador del conocimiento científico. Lo anterior tiene como base la IEP, para que el estudiante por indagación logre explorar y descubrir nuevos conocimientos. Los estudiantes mostraron gran interés y extrapolaron esta experiencia a sus hogares, donde asumieron el papel de investigadores y adoptaron la actividad en casa con resultados positivos.

En los marcos de las observaciones anteriores, se busca que en las instituciones educativas se enseñen con estrategias pedagógicas que contribuyan a las prácticas en conocimientos agrícolas, requiriendo que docentes y estudiantes concienticen que son capaces de producir nuevos procesos teóricos y prácticos que promuevan en ellos una motivación por aprender a aprender conceptos, identificar principios y tendencias de fenómenos, mediante la observación y el manejo directo.

Es importante que en cada uno de los estudiantes desarrolle la comprensión científica del contenido en su cotidianidad y en sus experiencias educativas específicas, en la actividad espontánea sobre las plantas; en lugar de conseguirla a partir de la explicación previamente organizada por los docentes. El hábito y las técnicas aprendidas por parte de los estudiantes con nuevas prácticas de cultivo, como la hidroponía, permite tener una visión más amplia de las habilidades que facilitan la puesta en marcha de lo estudiado y la investigación de otros métodos no habituales, que admite el cultivo de plantas en un medio que es libre de suelo.

Es necesario que se genere la enseñanza de las nuevas técnicas agrícolas, puesto que se debe encaminar en el estudiante el desarrollo de la capacidad para enfrentar diferentes situaciones, de tal modo que se puedan aplicar las experiencias ya adquiridas y que a su vez se permitan buscar 
otras que puedan ser beneficiosas, o en su defecto produzcan nuevos conocimientos mediante la investigación científica de aquellos problemas que se vayan afrontando.

Sobre la base de las consideraciones anteriores, el quehacer didáctico de las ciencias toma las situaciones del ambiente como principal actor para la enseñanza y el aprendizaje, y por esto se logra articular espléndidamente con los elementos integradores de la Educación Ambiental, aplicada desde la transversalidad de las áreas académicas de la institución educativa. Es por esto que una guía clara la tenemos en la Educación Ambiental, que ofrece objetivos y principios teórico-metodológicos basados en una postura interdisciplinaria y en la adopción de metodologías de diversas ciencias en cuanto a la comprensión y resolución de las complicaciones ambientales. Por lo tanto, se asume como principio básico que el ambiente es un recurso natural que debe ser revelado, interpretado y apreciado.

En ese mismo sentido, los argumentos anteriormente descritos tienen mucha importancia para la enseñanza y el aprendizaje de las ciencias experimentales, y concretamente para el caso de las nuevas técnicas donde se busca que los jóvenes tengan la ocasión de verse ellos mismos como intelectuales y que se propicie la participación motivadora en el hallazgo de los últimos adelantos científicos; llevados a cabo para optimizar la producción de cultivos y alimentos. Al interactuar activamente con sus maestros en actividades afines con su forma de vida y sus conocimientos y si se mantiene esta interrelación en el tiempo, puede servir como un impulso para el crecimiento de los investigadores agrícolas del mañana.

En el orden de las ideas anteriores, surge un gradual interés por buscar nuevas formas de enseñar y de aprender, en el cual se genere el desarrollo de competencias bá- sicas en los estudiantes, y se logre emplear mayor atención de la que se está prestando a la composición de nuevas experiencias de aprendizaje fuera del aula, es decir con mecanismos para complementar o potenciar la enseñanza formal, debido a que se requieren métodos prácticos y teóricos que susciten en el estudiante un interés por aprender conceptos, identificar nociones y tendencias de fenómenos que, a partir de la observación y el manejo directo, los estudiantes amplíen la comprensión del contenido por su propio interés en actividades investigativas sobre las plantas, en lugar de obtenerla a partir de la explicación previamente organizada por los docentes del área en la institución educativa.

Es evidente entonces, que el estudio de la Biología más específicamente en la botánica o agronomía debe generarse a partir de una experiencia concreta, donde se permita involucrar por completo, claramente y sin preocupaciones a los estudiantes en nuevas experiencias, haciendo uso del material a trabajar concisamente, es decir que no existan limitaciones con la utilización de dibujos, con los esquemas florales o en su defecto en la observación de ejemplares en los jardines de la escuela en los que ellos puedan manipular. Partiendo de esto se genere en el estudiante el proceso investigativo el cual debe contener la construcción de preguntas, y ciertos espacios abiertos para la socialización y la reflexión acerca de los resultados obtenidos, es decir que permitan no solo el aprender haciendo sino también el aprender reflexionando, lo cual llevará a los implicados a que se fomente al análisis racional de las ideas postuladas, los conocimientos trabajados y aprendidos, para así poder actuar bajo la capacidad en que se comprendan las situaciones a fin de que estos consigan dominar los conceptos, teorías, procedimientos, métodos, y que además de esto los estudiantes logren desarrollar actitudes y que practiquen sus valores. 
En ese orden de ideas, para conseguir un mejor beneficio de las ciencias es necesario que en los estudiantes esté presente la investigación desde las aulas educativas y en este caso, los cultivos hidropónicos, porque son una excelente estrategia pedagógica que permitirá trabajar en ellos las competencias desde el aprender haciendo, se trabaja la técnica que se pueda aplicar en la escuela y en la casa, y se promueve activamente la conciencia ambiental y el respeto por el medio ambiente.

Un aprendizaje importante de esta experiencia de trabajo es que la transformación de las prácticas pedagógicas, que estimulan a los estudiantes a que se apropien y hagan uso de la investigación, como fuente o medio para encaminar su tiempo libre en los contextos, desarrolla habilidades para el proceso de la observación, la experimentación, la investigación y la creatividad, permite estar consciente de los avances en los logros planteados y genera satisfacción en el docente por el alcance de las metas cumplidas, las cuales se hacen evidentes en las evaluaciones a nivel institucional.

Este trabajo definió caminos para fomentar la realización de comportamientos más respetuosos con el medio ambiente, lo que es muy importante, porque el progresivo deterioro del medio y sus consecuencias avizoran también el deterioro de la calidad de vida de la ciudadanía. Este escenario demanda la toma de conciencia y el compromiso individual y colectivo con acciones educativas que hagan sostenible el comportamiento con sensibilización medioambiental

\section{Referencias}

Arquigráfico. (2018). Hidroponia o Cultivos Sin Tierra - Definicion, Usos y Ventajas. Arquigrafico. Recuperado de https://arquigrafico.com/hidroponia-ocultivos-sin-tierra-definicion-usos-yventajas/
Báez y Pérez, J. (2009). Investigación cualitativa. Madrid: ESIC Editorial.

Beltrano, J. y Gimenez, D. (2015). Cultivo en hidroponía. La Plata: D - Editorial Universidad Nacional de La Plata.

Cajiao, J. y Uribe, P. (2016). Los cuatro pilares de la educación para el siglo XXI. Magisterio. Recuperado de https:// www.magisterio.com.co/articulo/loscuatro-pilares-de-la-educacion-para-elsiglo-xxi

Delors, J. (1994). Los cuatro pilares de la educación. La Educación encierra un tesoro. México, D.C.: El Correo de la UNESCO.

Fontán, Q. (2016). ¿Qué son los cultivos hidropónicos?. El Sol. Recuperado de https://www.elsol.com.ar/que-son-loscultivos-hidroponicos.html

Grijota, E. (2017). Cómo plantar tus alimentos en casa y sin usar tierra, lo próximo que todos querrán hacer. La Vanguardia. Recuperado de http:// ww w.lavanguardia.com/vivo/nutricion/20170125/413630299065/que-esel-cultivo-hidroponico-ventajas-inconvenientes.html

Hernández, R., Fernández, C. y Baptista, P.(2014).Metodologíadelainvestigación. México, D.F.: McGraw-Hill.

Martínez, M. (2004). Ciencia y arte en la metodología cualitativa. México D.F: Trillas

Mejía, M. y Manjarrés, M. (2011). La investigación como estrategia pedagógica una apuesta por construir pedagogías críticas en el siglo XXI. Praxis \& Saber, 2(4), $127 . \quad$ http://dx.doi. org/10.19053/22160159.1127

Orozco, M. (2015). Métodos, formas, procedimientos y sistemas de enseñanza en una concepción de la pedagogía como arte según José M. Zamora. Aula, 21(0), $165 . \quad$ http://dx.doi.org/10.14201/ aula201521165176 
Pérez, G. (2002). Investigación cualitativa. Retos e interrogantes. II técnicas y análisis de datos. Madrid: la Muralla S.A.

Restrepo, B. (2009). Investigación de aula: formas y actores. Bibliotecadigital. udea.edu.co. Recuperado de http://bibliotecadigital.udea.edu.co/bit $\mathrm{str}$ ea m/10495/3498/1/ RestrepoB ernardo_2 009 Investigaci\%C3\%B3naulasformas.pdf

Ruiz, J. (2012). Metodología de la investigación cualitativa. Bilbao: Universidad de Deusto.
Strack, J., Suárez, J., Di Mauro, G., \& Jacob, S. (2014). Impacto de la iluminación residencial eficiente en la calidad de la energía de una red de distribución. INGE CUC, 10(2), 9 - 19. Recuperado de https://revistascientificas.cuc.edu.co/index.php/ingecuc/article/view/485

Villalba, A. (2012). Dos paradigmas contrapuestos en la evaluación educativa, dos formas diferentes de concebir al alumno. Aula Universitaria, 1(14). http://dx.doi.org/10.14409/au.v1i14.4123 